\title{
Some applications of enthalpimetric detection in flow injection analysis
}

\author{
W.E. van der Linden, M. Bos, H.H. Heskamp, and H. Wilms \\ Department of Chemical Technology, University of Twente, NL-7500 AE Enschede, The Netherlands
}

\section{Einige Anwendungen der enthalpimetrischen Detektion in der Fließinjektionsanalyse}

Summary. A flow injection manifold is described based on a flow-through arrangement of thermistors for measuring heat effects of chemical reactions. Temperature changes down to $10^{-3}{ }^{\circ} \mathrm{C}$ can be measured reproducibly. The working range for the determination of a component is dependent on the reaction enthalpy and can vary from $10^{-3}$ to $10 \mathrm{~mol} / 1$ for acids and bases, from 10 to $1000 \mathrm{ppm}$ for e.g., alkylhydroperoxides. Sample frequencies up to 150 samples per hour can be realized.

\section{Introduction}

Some years ago Pasquini and de Oliveira [1] published a paper on the enthalpimetric detection in flow injection analysis. At that time a similar investigation was going on in our laboratory, but the results have not been published because essentially the same conclusions were reached as far as the methodological aspects are concerned.

However, the paper presented only one simple example and therefore may have failed to draw the attention it deserves. It seems appropriate to present some more examples in order to illustrate the potentialities of the enthalpimetric approach, and to stress some experimental aspects in more detail.

The main advantages of enthalpimetric flow-trough detection are:

- the general applicability;

- the robustness of the device;

- the corrosion-resistivity of the glass-coated thermistors used, which allows the construction of small systems suitable for rather aggressive media;

- the fact that adsorption of components does not directly affect the analytical results unless the detector response has become so sluggish that it will become the determining factor in the overall signal generating process.

A possible drawback of the device might be that great care has to be taken to avoid noise and drift. Therefore, the temperature of the solutions should be exactly equilibrated prior to the reaction and the basic composition of carrier stream and reagent stream(s) should be such as to minimize heat effects on mixing. Furthermore, the influence of flow

Offprint requests to: W.E. van der Linden rate fluctuation has to be eliminated. This can be achieved by means of a differential measuring system in which two thermistors are used in a Wheatstone bridge arrangement. One thermistor is situated at the end of the adiabatic reaction coil and the reference thermistor is placed in the same stream after passing a stainless steel heat exchanging equilibration coil [2].

\section{Experimental}

\section{Chemicals}

All chemicals were of analytical reagent grade. In the determination of peroxides and hydroperoxides commercially available solvents for Karl Fischer determinations were used as the $\mathrm{SO}_{2}$-containing reagent (KF solution $\mathrm{A}$, Merck and Hydranal solvent, Riedel del Haën), as well as a solution containing $100 \mathrm{~g}$ imidazol (purum, Fluka $\mathrm{AG}$ ) in 11 water saturated with $\mathrm{SO}_{2}$ (about $1.6 \% \mathrm{SO}_{2}$ ). Before use the latter solution was diluted ten times and stored under nitrogen.

\section{Manifold}

Figure 1 shows the flow injection system as well as the measuring device. The system is essentially similar to the one suggested by Pasquini and de Oliveira [1] for the merging zones approach. The major part of the manifold is immersed in a thermostatted water bath. Pulse dampers in both carrier

Table 1. Equipment used for construction of manifold and measuring device

\begin{tabular}{lll}
\hline Part & Manufactures & Type \\
\hline Waterbath & Tamson & T. E. 3/150 \\
Strip-chart recorder & Kipp & B.D. 8 \\
Persiataltic pump & Gilson & Minipuls 2 \\
Pumb tubing & Gilson & Several diameters \\
Tube connectors & Rheodyne & - \\
Injection valve & Rheodyne & 5020 A \\
Equilibrating coils & Chrompack & 4005 S.S. $1 / 16^{\prime \prime}$ \\
& & (stainless steel $0.5 \mathrm{~mm}$ \\
& & i.d. and $1.6 \mathrm{~mm}$ o.d.) \\
Other conduits & - & Teflon $0.5 \mathrm{~mm}$ i.d. \\
Thermistors & Philips & 2322 627 42223 \\
Voltage source & Methrohm & E 371 \\
Amplifier & Knick & Hv \\
\hline
\end{tabular}




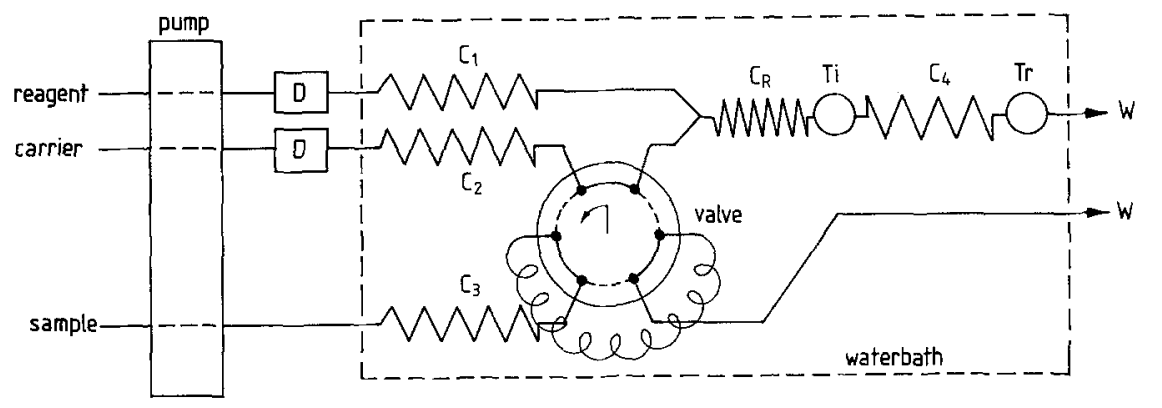

D : pulse dampener

$C_{1}-C_{4}$ : equilibration coils

$C_{R} \quad$ : reaction coil

$\mathrm{T}_{i} \quad$ : indicator thermistor

$T_{\Gamma} \quad$ : reference thermistor

W : waste

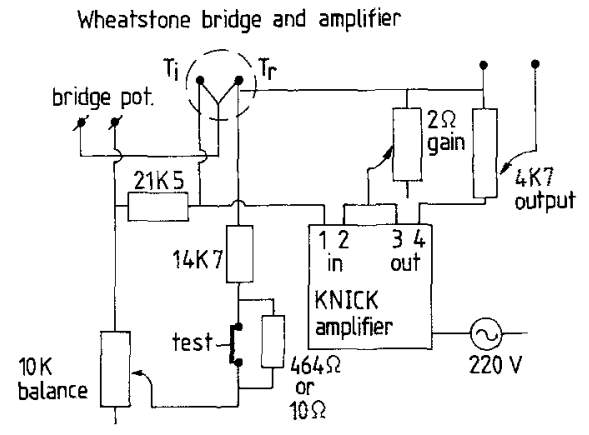

Fig. 1

Schematic diagram of enthalpimetric flow injection system and measuring device and reagent lines are constructed from a T-piece connector together with a disposable injection syringe; they are strongly recommendable because of the flow dependence of the thermistor responses. The reaction coil consisted of a $0.55 \mathrm{~mm}$ i.d. and $1.5 \mathrm{~mm}$ o.d. teflon tube wrapped in aluminum foil and packed in a PVC-tube of $2.3 \mathrm{~mm}$ i.d. to ensure approximately adiabatic conditions. Further instrumental information is summarized in Table 1.

In one of the arms of the Wheatstone bridge a small additional resistance $\left(R_{\text {test }}\right)$ is inserted. The scale deflection $\left(U_{0}\right)$ obtained on shortcircuiting this resistance can be used to calibrate the scale in centigrades using the equation:

1 scale unit $=R_{\mathrm{test}} / \alpha R_{\mathrm{th}} U_{\mathrm{o}}$

where $\alpha$ is the negative temperature coefficient (typically $-4 \% /{ }^{\circ} \mathrm{C}$ for the thermistors used here) and $R_{\mathrm{th}}$ is the resistance of each thermistor $\left(R_{\mathrm{th}}=21.5 \mathrm{k} \Omega\right)$.

The applied voltage for the bridge was $100 \mathrm{mV}$.

\section{Results and discussion}

\section{Instrumental aspects}

The logarithmic value of the resistance of a thermistor is linearly dependent on the temperature:

$\ln R_{\mathrm{th}}=\alpha T$

with $\alpha$ as the negative temperature coefficient.

For small temperature changes this equation can be simplified to:

$R_{\mathrm{th}}=R_{\mathrm{th}, \mathrm{o}}+\alpha R_{\mathrm{th}, \mathrm{o}} \Delta T$

where $R_{\mathrm{th}, \mathrm{o}}$ is the resistance at $T_{\mathrm{o}}$, and $\Delta T=T-T_{\mathrm{o}}$.

Provided that in all four branches of the Wheatstone bridge the resistance is about $R_{\mathrm{th}, \mathrm{o}}$ (ca. $21.5 \mathrm{k} \Omega$ ) than it can be derived that:

$V_{\mathrm{m}}=\frac{\alpha V_{\mathrm{o}}\left(\Delta T_{\mathrm{r}}-\Delta T_{\mathrm{i}}\right)}{4+2 \alpha\left(\Delta T_{\mathrm{r}}-\Delta T_{\mathrm{i}}\right)} \approx \frac{\alpha V_{\mathrm{o}}}{4}\left(\Delta T_{\mathrm{r}}-\Delta T_{\mathrm{i}}\right)$.

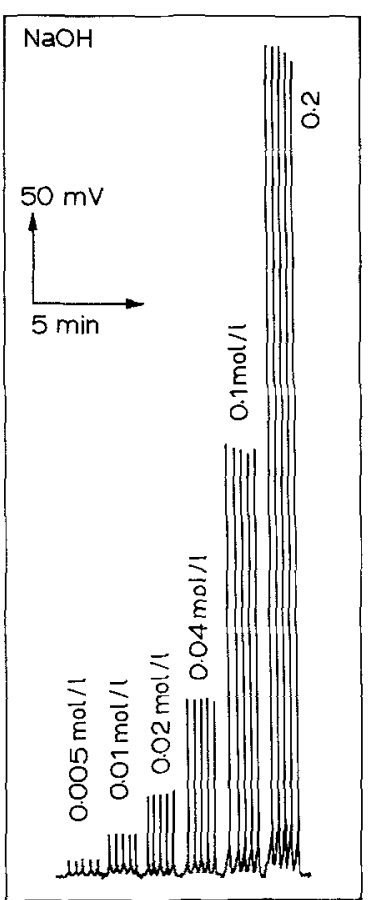

Fig. 2. Signals obtained for various concentrations of $\mathrm{NaOH}$; reagent $\mathrm{HCl}$

$V_{\mathrm{o}}$ and $V_{\mathrm{m}}$ refer to the applied and measured voltages, respectively, whereas $\Delta T_{\mathrm{r}}$ and $\Delta T_{\mathrm{i}}$ are the temperature changes at the corresponding thermistors. So, the measured voltage will exhibit a linear relationship to the temperature difference between both thermistors.

The sensitivity of the detection system could be enhanced by increasing the bridge voltage but this is accompanied by two deleterious effects: first, an increase of noise of the 


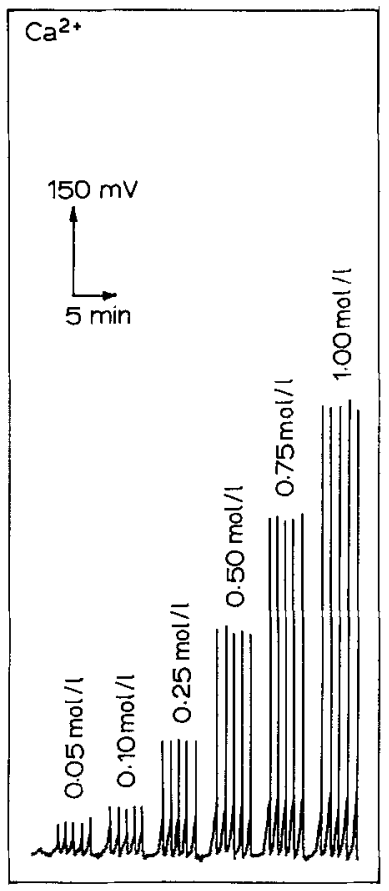

Fig. 3. Signals obtained for various concentrations of $\mathrm{Ca}^{2+}$; reagent EDTA, buffer $\mathrm{NH}_{3} / \mathrm{NH}_{4} \mathrm{Cl}, \mathrm{pH} \approx 10$

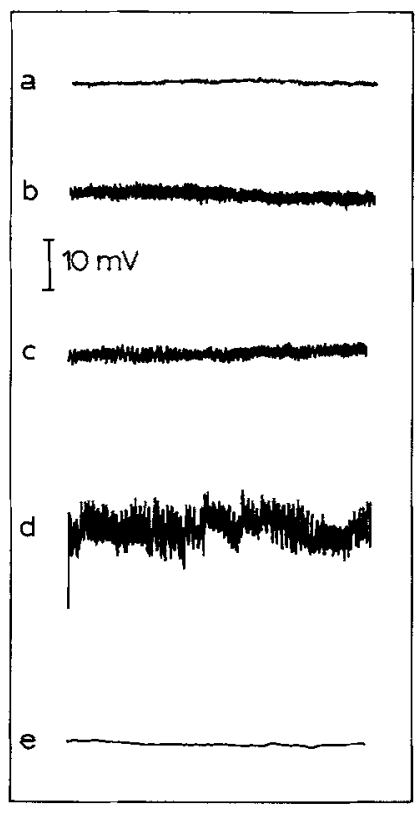

Fig. 4. Effect of composition of solvents on the noise of the base line; carrier stream is water, reagent stream is: $a$ Hydranal/water $=1 / 10 ; b$ Hydranal $/$ water $=1 / 5 ; c \mathrm{KF}$-solution A (Merck) $/$ water $=1 / 5 ; d \mathrm{KF}$-solution $\mathrm{A}($ Merck $) /$ water $=1 / 2 ; e \mathrm{SO}_{2} /$ imidazol $/$ water

thermistors is observed and, secondly, the selfheating of the thermistors may become appreciably. For a spherical thermistor this self-heating effect can be estimated by means of the following expression:

$\Delta T=\frac{V_{\mathrm{o}}^{2}}{16 \pi \lambda R_{\mathrm{th}}}\left(\frac{1}{r}-\frac{1}{r+\delta}\right)$ where $\Delta T$ is the temperature difference between interior and exterior, $r$ is the radius of the thermistor interior, $\delta$ is the thickness of the glass surrounding the interior and $\lambda$ is the thermal conductivity of glass. For the thermistors used $(r=$ $0.5 \mathrm{~mm}, x=1 \mathrm{~mm}, \lambda=1 \mathrm{~W} \cdot \mathrm{m}^{-1} \cdot \mathrm{K}^{-1}, R_{\mathrm{th}}=21.5 \mathrm{k} \Omega$ ) $\Delta T$ is approximately $1 \cdot 10^{-3} \cdot V_{0}^{2}$, so for the applied $100 \mathrm{mV}$ this leads to a temperature difference $\Delta T \sim 10^{-5}{ }^{\circ} \mathrm{C}$ and the self-heating effect may be neglected in most cases.

For an enthalpimetric flow-through detector a detector efficiency, $E_{\mathrm{d}}$, can be defined:

$E_{\mathrm{d}}=\frac{\Delta T_{\text {measured }}}{\Delta T_{\text {ideal }}}$.

The value of this efficiency is influenced by heat transference to the wall of the reaction coil, the response time of the thermistors and the degree of conversion which depends on both kinetic and hydrodynamic factors as well as the geometry of the cell. Typically, efficiencies of close to $90 \%$ were found; no attempt was made to increase this value any further

\section{Acid-base reactions}

Some results for the determination of $\mathrm{NaOH}$ in the range of $5 \cdot 10^{-3}$ to $2 \cdot 10^{-1} \mathrm{~mol} / 1$ are shown in Fig. 2. The detection limit is about $10^{-3} \mathrm{~mol} / 1$ corresponding to a temperature change of about $10^{-3}{ }^{\circ} \mathrm{C}$. The concentration of the reagent stream should be adapted to some extent to the concentration range of the analyte. This means that in the higher concentration range (e.g., $1-10 \mathrm{~mol} / 1 \mathrm{NaOH}$ ) a higher baseline together with an increased noise level is observed due to the heat evolved on sheer mixing of reagent and carrier stream. Nevertheless, an upper limit of about $10 \mathrm{~mol} / \mathrm{l}$ could be reached with results of adequate accuracy. It may be clear that the enthalpimetric detection cannot compete with the $\mathrm{pH}$ glass electrode in the lower concentration range but, especially in the monitoring of streams of concentrated acids and bases, it will offer good possibilities. Quantitative results are summarized in Table 2.

\section{Complexometric reactions}

The enthalphy of the reaction of calcium with EDTA is rather low resulting in a detection limit of about $10^{-2} \mathrm{~mol} / \mathrm{l}$. The upper limit was found to be $2 \mathrm{~mol} / \mathrm{l}$ bound to the limited solubility of EDTA and the increased viscosity of more concentrated EDTA solutions. An increase in temperature to about $45^{\circ} \mathrm{C}$ in order to circumvent these problems caused gas bubble formation in the system probably because of the ammonia. No attempts were made to look for other buffers because there seems to be hardly any need for the determinations of such high calcium concentrations. A calibration series is depicted in Fig. 3; results are summarized in Table 2.

\section{Determination of hydrogen peroxide and alkylhydroperoxides}

Hydrogen peroxide and alkylhydroperoxides can be quantitatively reduced by sulphur dioxide in the presence of pyridine or some other buffering amine. The reaction enthalpy is quite high, allowing determinations in the ppm-range. Commercially available solutions prepared for the Karl Fischer determination can be used as the reagent, but special attention has to be paid to matching the basic solvents used in carrier and reagent stream in order to avoid large heat 
Table 2. Quantitative results of standard series of various components

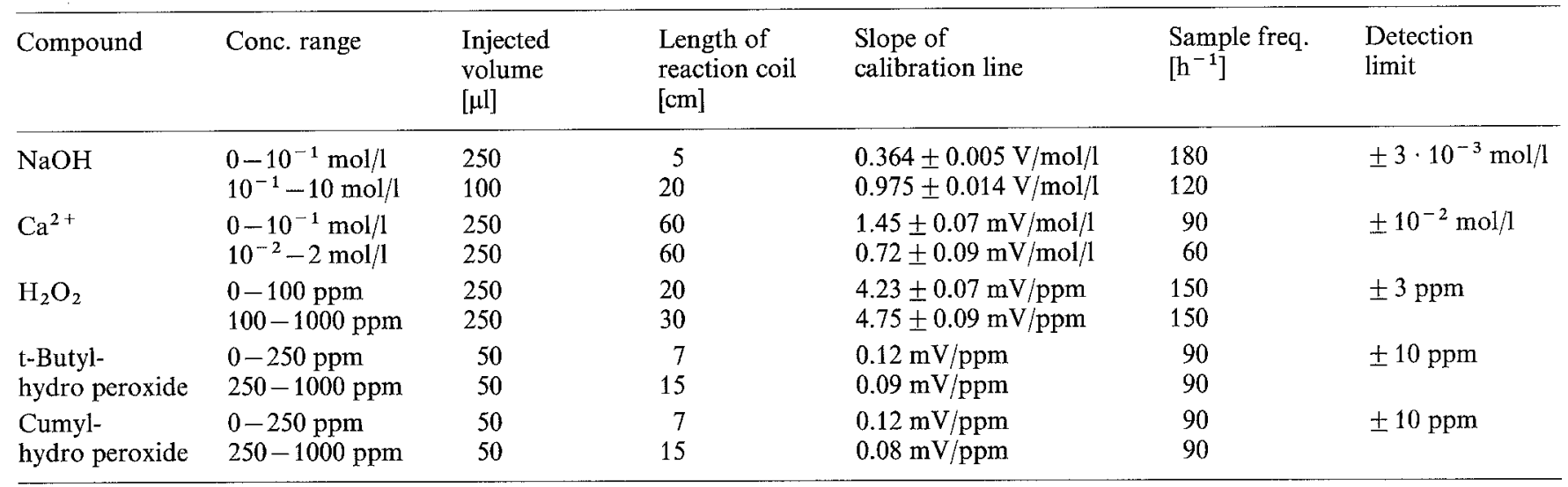
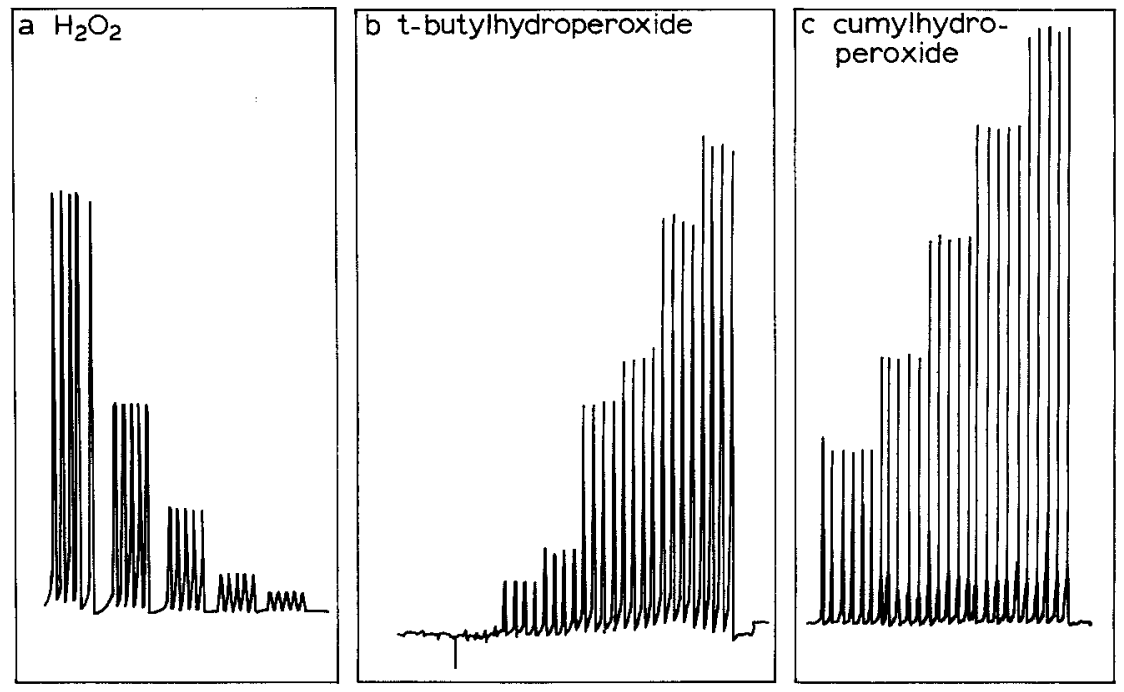

Fig. 5a-c

Signals obtained for hydrogen peroxide (a), t-butylhydroperoxide (b) and cumylhydroperoxide (c)

effects due to sheer mixing. In Fig. 4 this effect is illustrated for the reaction of aqueous $\mathrm{H}_{2} \mathrm{O}_{2}$ samples where water is the carrier stream and reagent streams of various compositions have been used. Obviously the $\mathrm{SO}_{2} /$ imidazol $/ \mathrm{H}_{2} \mathrm{O}$ reagent exhibits the least noise, and therefore it was used for the determination of hydrogen peroxide. For the determination of t-butylhydroperoxide in the range $0-250 \mathrm{ppm}$ the reagent consisted of a KF-solution ( $\mathrm{SO}_{2} /$ pyridine/methanol) which was ten times diluted and the carrier stream was a $10 \%$ aqueous methanol solution. On the other hand, for cumylhydroperoxide which is soluble in methanol and toluene, methanol was used as the basic medium for both reagent and carrier stream. In Fig. 5 some typical series of peaks are shown; quantitative results are summarized in Table 2.

\section{Conclusions}

Enthalpimetry has been shown to be a suitable technique for the detection of various types of reactions. Provided that sufficient attention is paid to an adequate design of the manifold, temperature changes as low as $10^{-3}{ }^{\circ} \mathrm{C}$ can be measured reproducibly. This leads to detection limits that depending on the reaction enthalpy - can range from $10^{-2} \mathrm{~mol} / \mathrm{l}$ down to the ppm-level. A major advantage, however, seems to be the applicability in the high concentration range, for instance for the determination of acid or base streams in process industry.

\section{References}

1. Pasquini C, de Oliveira WA (1984) Anal Chim Acta 156:307213

2. Schifreen RS, Miller CS, Carr PW (1979) Anal Chem $51: 278-$ 283

Received June 5, 1987 